in intensity as we pass from either end towards the middle of the group, and we may depict the relation of the two groups by drawing the enveloping curve for the region of absorption and for that of fluorescence and showing the extent to which they overlap, as in Fig. 4. While detailed quantitative studies of the absorption bands have not yet been made, the preliminary observations indicate that the enveloping curve is an "image" of that of the group of fluorescence bands, just as the broad absorption band of resorufin and other fluorescent substances has already been $\operatorname{shown}^{16}$ to be the overlapping image of the corresponding fluorescence band.

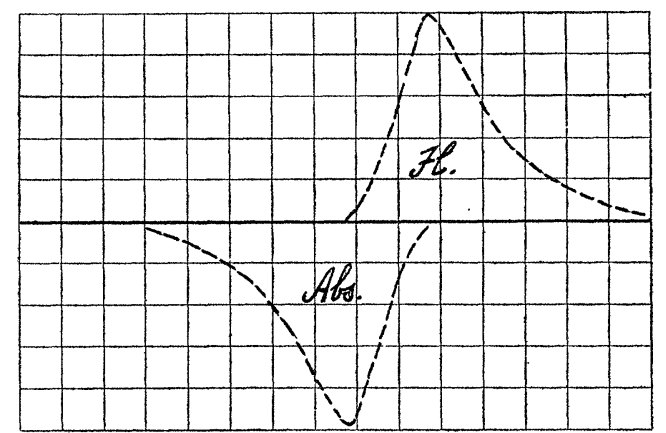

Fig. 4. Diagram showing the overlapping of fluorescence and absorption in the case of a uranyl salt.

Not only is the envelope of the fluorescence group of the same type as the curve of distribution of energy in ordinary fluorescence bands, but each component, as has been shown by spectrophotometric measurements, has a similar distribution of energy. Moreover, both curves have the same characteristics, although with very different scales of wave-length, as the energy curve of the temperature radiation of a black body. That the relation between the luminescence of solids and liquids and that of vapors is an intimate one is strongly suggested by the comparison of the diagram from Steubing's measurements of the fluorescence of oxygen (Fig. 1) with the curves for the uranyl salts just described, and this suggestion is strengthened by the inspection of

${ }^{16}$ Nichols and Merritt, Physical Review, Vol. XXXI., p. 376.
Wood's curves for the fluorescence of sodium vapor. Moreover, the family resemblance of all these related cases of banded fluorescence spectra to the banded spectra of gases as we find them described in the papers of Deslandres and of Kayser and Runge is unmistakable. In view of these numerous indications of a common property may we not anticipate the attainment of some broader generalization concerning the various types of radiation than has hitherto been made?

E. L. NiCHOLS

\section{NOTES ON THE PRELIMINARY REPORT OF \\ THE COMMITTEE ON THE TEACHING \\ OF MATHEMATICS TO STUDENTS \\ OF ENGINEERING}

Ar the meeting of the Society for the Promotion of Engineering Education held at Madison, Wis., in June, 1910, the members present were handed sets of galley proofs of the Preliminary Report of the Committee on the Teaching of Mathematics to Students of Engineering, which committee was appointed at a joint meeting of the American Mathematical Society and Sections A and D of the American Association for the Advancement of Science in December, 1907. The committee consists of twenty members, ten of them being professors of mathematics, three presidents of technical institutions and seven professors of engineering and consulting engineers.

The report being only a preliminary one, it is subject to amendment before being presented as a final report, and no doubt the members of the committee will be glad to receive any suggestions which will tend to make the report more useful and more acceptable to engineering teachers. The writer acknowledges the great value of the report as a whole, but he has some criticisms to offer on one portion of it, viz., elementary dynamics, which he hopes will be carefully considered by the members of the committee and by others interested, who may be led by it to offer the committee additional suggestions.

In the preface to the report it is said:

The defects in the mathematical training of the student of engineering appear to be largely in knowledge and grasp of fundamental principles, 
and the constant effort of the teacher should be to ground the student thoroughly in these fundamentals, which are too often lost sight of in a mass of details.

The defects mentioned are universally admitted and complained of, and they are perhaps more apparent in the subject of elementary dynamics than in any branch of pure mathematics, but the writer is of the opinion that they are due rather to the text-books than to the teachers. As the committee says: "What is most needed at the present time is a series of synoptical text-books which shall present in compact form (1) the fundamental principles of the science, and (2) a classified and graded collection of problems."

The writer heartily commends the two following sentences in the report:

The poundal is never used in practise, and no instruments are on the market which give readings in poundals; it should be dropped from the textbooks. The so-called "engineers' units of mass," namely, the G-kilogram, or "'metric slugg," and the g-pound, or "'slugg," are never used in practise, and no set of weights is on the market giving readings in terms of these units; they should be dropped from the text-books.

The chief trouble with the text-books is due to the fact that some early writer, in his attempt to overcome what he conceived to be a difficulty in teaching the subjects of weight and mass, invented the "poundal," and others invented the "engineers' unit of mass," "geepound" or "slugg" (32.2 pounds of matter), to overcome the imaginary difficulty, and other text-book writers blindly followed them. These devices did not overcome the difficulty, but on the contrary only created confusion, and students had to spend weary hours on these worse than useless units, which afterwards, when they became engineers, they had to unlearn.

The idea of the "poundal" is probably a development of the "C.G.S." system, in which 1 dyne (force) acting on 1 gram (matter) free to move, for 1 second, gives it a velocity of 1 centimeter per second. In English units, if 1 pound force acts for one second on 1 pound of matter, the velocity at the end of the time is 32.2 feet per second. If the English system only had either a unit of force $=1 / 32.2$ pound, or a unit of quantity of matter $=32.2$ pounds, then the figure 32.2 in the equation of the relation of force to acquired velocity would disappear, and the equation would look simpler; so the two new units, poundal and gee-pound, were invented, to the confusion of the subject, and they now have to be expunged from the language.

Merely getting rid of the poundal and the gee-pound, however, does not get rid of the whole trouble arising from the use of the same unit, pound, to express both a force and a quantity of matter. The committee (or perhaps only one or two members of it, to whom were assigned the subject of dynamics) have wrestled with the problem, but in the writer's opinion they have failed to find the best solution of it.

In order to make this criticism as clear as possible, the following extracts are first made from the committee's report, and comments on the several paragraphs follow.

\section{Extracts from the Report}

(a) The most important mechanical quantities for engineering purposes are length, time, angle and force. Derived from these are: area, volume, pressure, linear velocity, etc.

(b) Less important for the engineer, but of great importance in general scientific work, is the quantity called "mass" (galley 39).

(c) The units of mass are of little importance to the engineer, since the quantity that enters into the equations of engineering is not the mass of the body, in units of mass, but its normal weight, in units of force.

(d) The mass of a body may be thought of, roughly, as the amount of matter in a body (galley 40).

(e) The ratio $W / g$ for a given body is constant in all localities. This quantity, or a quantity proportional to this ratio, is called the mass, $m$, of the body (galley 35).

( $f$ ) The weight, $W$, of a body, at a given place, is the force that causes it to fall when unsupported (galley 34).

$(g)$ If the mass of a body is one pound 
(mass) then its normal weight is one pound (force) (galley 35).

(h) The normal weight, $W_{0}$, of a body is its weight in vacuo, at sea level in latitude $45^{\circ}$.

(i) A "set of standard weights" is a set of metal pieces each of which is marked with its normal weight.

$(j)$ The process of weighing a body on an equal arm balance, against a set of standard weights, gives the normal weight of the body.

(k) The process of weighing a body on a spring balance, on the other hand, gives the local weight of the body.

(l) To graduate the scale of a spring balance find the local weight of a set of standard weights as just explained (by computation from the value of $g$ at the locality in question) and then suspend the pieces successively on the spring, and mark on the scale the deflection caused by the local weight of each piece (galley 34 ).

$(m)$ Force may be thought of, roughly, as a push or a pull.

(n) $F / W=A / g$ is taken as the fundamental equation.

(o) If in the fundamental equation $F / W=$ $A / g$ we substitute the equation for mass, $m=c(W / g)$, where $c$ is the numerical factor depending on the choice of units, we have $c F=m A$. Any system of units which makes $c=1$ in this equation is called an absolute system of units.

( $p$ ) The equation $F=m A$ will give incorrect results if the forces and masses are given in any but absolute units. In particular it should never be used in the ordinary problems of engineering.

(q) On account of the special character of this equation $F=m A$ it is unfortunate that it should be so often taken as the fundamental equation of dynamics instead of the general equation $F / W=A / g$ from which it is derived (galley 41).

\section{Comments on the above Extracts}

(a) For "length, time, angle and force," we would better read "space, time, matter and force." Mechanics is the science of the action of force upon matter. Matter is that upon which force acts, and it is just as important as force in most engineering problems. Matter occupies space, therefore space is one of the four elementary concepts of mechanics. Length is merely the linear measurement of space in one direction, and therefore it should be placed in the list of derived quantities, with area and volume. Angle should also be placed in the list of derived quantities; it is the difference in direction of two lines that meet in a point, or the difference in position of a line that is rotated a certain distance about one of its ends.

$(b, c)$ If "the quantity called mass" is the quantity of matter in a body, then this statement is incorrect. The quantity of matter in a body is of the utmost importance to the engineer, independent of the force of gravity acting upon it, if the body is to be moved horizontally, or rotated, or accelerated, or if he or his client has to pay for it.

$(d, e)$ Here are two definitions of "mass" which are inconsistent. If mass is the quantity of matter, why should it be "thought of, roughly"? Why is it not defined with precision as the quantity of matter as determined accurately by weighing it on an even balance scale and compared with the standard pound (or kilogram)?

The second definition is the ratio $W / g$, or a quantity proportional to this ratio.

The fact is that the word "mass," as applied to matter, is used in three different senses:

1. As synonymous with "portion," "piece" or "lump" of matter, as in the expression "this mass weighs ten pounds," the words "weighs ten pounds" referring to the quantity of matter as determined by weighing.

2. As synonymous with the word "weight" (quantity of matter), as the word weight is universally used in ordinary language, and nine times out of ten by the engineer, who rarely uses the word "mass" in this sense. Example: "This lump has a mass of ten pounds."

3. As synonymous with the ratio $W / g$, where either $W$ is the weight (quantity of matter) and $g$ is 32.174 , the acceleration due 
to gravity at the sea level at latitude $45^{\circ}$, or $W$ is the force with which gravity attracts the body at any given place and $g$ is the acceleration due to gravity at that place. The quantity or ratio $W / g$ is constant in either case. In this the mass of the ten-pound lump is 10/32.174, and since it is a ratio it has no unit. The statement that "the engineers' unit of mass is 32.2 pounds," found in some books on physics, is incorrect. The engineer has no such unit.

Giving the name "mass" to the ratio $W / g$ was perhaps unfortunate, but it can not now be helped, since it is universally used in this sense in the engineering text-books of the past sixty years or more. It is a handy term, and the use of the single letter $M$ instead of the fraction $W / g$ often simplifies calculations. It is hard enough to get rid of a bad term, for example, "poundal," which has been in the high-school books on physics for the past thirty years in spite of the numerous attacks upon it. It is not likely that we can get rid of the term mass, $M$, in the sense of $W / g$ unless some one invents another and a better name for it.

Of course confusion results from the three different meanings given to the same word "mass," but the confusion arises chiefly from its use in the second sense given above, as synonymous with weight (quantity of matter). It is so used in all the books on physics, but as already stated, is rarely so used by engineers. It would tend to diminish the confusion if the books on physics mentioned that in engineering "mass" means the ratio $W / g$, and not the quantity of matter in pounds, thus preparing students for what they will afterwards learn in their engineering studies.

(f) "The weight, $W$, of a body, at a given place, is the force that causes it to fall if unsupported."

The word "weight" is used in two senses: (1) in the sense given in the above definition, which is that of the text-books on physics, and (2) in the sense of quantity of matter as determined by the common method of weighing it. In this sense it is used universally in ordinary literature and in commercial transactions, and nine times out of ten by engineers, in all calculations in which quantity of matter is involved.

In this sense $W$ is a constant quantity; in the first sense it is inconstant, varying with the latitude and with the elevation above the sea level.

In the second sense, quantity of matter, the word weight was used long before Newton's time. It is thus used in the clause of the constitution of the United States that authorizes congress to fix the standard of weights and measures, in acts of congress establishing the Bureau of Weights and Measures, and in acts of the British parliament. It is thus used in the King James version of the English Bible: "And they shall eat bread by weight" (Ezek. $4: 16$ ). It is not conceivable that this meaning of the word "weight" can ever go out of use. It is in the language of the people and it is there to stay.

The beginning of confusion in the minds of students as to the meaning of the words "weight" and "mass" results from the fact that the high-school text-books use the word mass for what is commonly called weight, and attempt to restrict the word weight to mean only the force with which a body is attracted by gravity at a given place.

$(g, h)$ "If the mass of a body is one pound (mass) then its normal weight is one pound (force)."

By mass we here understand quantity of matter, and not the ratio $W / g$ given in definition $(e)$.

The "normal weight" (this appears to be a new and useless term, and therefore undesirable) is the force with which a body is attracted to the earth by gravity at latitude $45^{\circ}$.

If the sentence $(g)$ were made to read: "If the weight of a body is one pound, then the force with which gravity attracts it at the sea level in latitude $45^{\circ}$ is one pound," it would be strictly accurate, in harmony with the every-day use of the language, and would avoid the confusion arising from the use of the word "mass." The meaning of the word "weight" in this sentence is not ambiguous 
or doubtful. It means both that the quantity of matter will balance the standard pound on an equal arm balance, and that the force which gravity exerts on it at latitude $45^{\circ}$ is one pound, as indicated by a properly graduated spring balance.

$(i, j)$ If for the words "normal weight" in these two sentences we use simply the word weight, it will express the idea accurately, whether the word means quantity of matter or the force with which gravity would act on the body at latitude $45^{\circ}$. The two meanings are synonymous and the two quantities identical. The measure of the quantity of matter in a body is the measure of the force with which gravity attracts it at latitude $45^{\circ}$.

(k) The process of weighing a body on a spring balance, on the other hand, gives the "local weight" of a body. The term "local weight" also appears to be new, but as it strictly expresses the idea of the attraction of gravitation on a body at a given locality, and there is no other short term that so clearly expresses it, it may be considered unobjectionable. The words "gravity of a body" might properly be used to express the idea, if textbook writers would agree to it, for its value would vary in the same proportion as the earth's gravitational force varies, with the locality.

The sentence $(k)$, however, is true only if the spring balance has been graduated with standard weights at latitude $45^{\circ}$. If graduated with standard weights at the locality (other than lat. $45^{\circ}$ ) where the weighing is done, it will give the "normal weight," or quantity of matter, just as an even balance would do.

(l) This sentence is not clear. A spring balance graduated, say, at latitude $30^{\circ}$, by hanging on it successively the standard weights, $1,2,3$, 4, etc., pounds, and marking the deflection shown by each, will show at latitude $30^{\circ}$ the weight (quantity of matter) of bodies weighed on it, but if it is desired that the balance should indicate "local weight," then the standard weights hung on it should be increased in the proportion that the attraction of gravitation is less at latitude $30^{\circ}$ than at latitude $45^{\circ}$, or in the ratio $32.174 \div 32.131$, or $1 / 1.0013$. If 1.0013 pounds is hung on the scale it will indicate 1 pound, and 1 pound hung on it will indicate 0.9987 pound, the "local weight" of 1 pound of matter at latitude $30^{\circ}$. Spring balances, however, are never used for weighing as accurately as 1.3 parts in 1,000 , since for such weighing a microscope would be needed to read the deflections. "Local weight" is rarely needed in engineering problems, and if it should be needed it is determined not by weighing on a spring balance but by multiplying the weight (quantity of matter) by the ratio of the value of $g$ at the location to 32.174.

(m) "Force may be thought of, roughly, as anything of the nature of a push or a pull." Why "roughly"? The definition of force as a push or a pull is as precise as language can make it.

(n) " $F / W=A / g$ is taken as the fundamental equation." This is only one form of the fundamental equation. The fundamental fact in dynamics is that if a force $F$ is exerted constantly for a time $T$ upon a body free to move, whose weight is $W$, giving it a velocity $V$ (starting from rest when $V=0$ ) at the end of the time $T$, then the following equation is true (in the foot-pound-second system of units) :

$$
F T=(W / g) V,
$$

or, if $A=V / T$ then $F g=W A$, from which the equation $F / W=A / g$ is derived; also if $M=W / g$, then $F T=M V$ and $F=M A$.

(o) "If in the fundamental equation $F / W=A / g$ we substitute the expression for mass, $m=c(W / g)$, where $c$ is the numerical factor depending on the choice of units, we have $c F=m A$. Any system of units which make $c=1$ in this equation is called an absolute system of units."

The value of $c$ in the foot-pound-second system is given as 32.174 , and in the dynegramme-second system as 1 . In the footpound system $m=32.174 \mathrm{~W} / \mathrm{g}$.

This is out of harmony with the engineering text-books and with engineering literature 
generally, which make $M=W / g$. If students are taught in elementary mechanics that $m=32.174 \mathrm{~W} / \mathrm{g}$ and in engineering that $M=W / g$, the resulting confusion will be as great as that caused by the poundal and the gee-pound.

(p) "The equation $F=m A$ will give incorrect results if the forces and masses are given in any but absolute units. In particular it should never be used in the ordinary problems of engineering."

The reasoning that leads up to this conclusion would also condemn the use of the time-honored formulæ $F T=M V$ and $F S=$ $1 / 2 M V^{2}$.

The equation $F=M A$ is given in all engineering text-books that deal with problems in dynamics, and it has been used from time immemorial with English units and with perfect accuracy.

(q) "On account of the special character of this equation, $F=M A$, it is unfortunate that it should be so often taken as the fundamental equation of dynamics, instead of the general equation $F / W=A / g$ from which it is derived."

As commonly used by engineers the equation $F=M A$ has no more a special character than the equations $F T=M V$ and $F S=$ $1 / 2 M V^{2}$. It is just as fundamental as $F / W=A / g$, which as well as $F=M A$ may be derived from $F T=M V$. If in the last equation we take $V / T=A$, then $F=M A$; and if $M=W / g$, then $F / W=A / g$.

Whether a certain equation should or should not be used in engineering depends, (1) on its logical correctness, (2) upon its usefulness. The three equations $F=M A, F T=M V$ and $F S=1 / 2 M V^{2}$, are all logically correct, equally in the so-called "absolute" (C.G.S.) system, the pound-foot system, and the kilogram-centimeter system, provided that in the C.G.S. system the unit of quantity of matter is the gram and the unit of force is the dyne, or $1 / 981$ of the force with which gravity attracts a gram of matter at latitude $45^{\circ}$; that in the pound-foot system $M=W / 32.2$ and that in the kilogram-centimeter system $M=W / 981, W$ being the weight of the body in pounds (or kilograms) and the definition of weight being the quantity of matter in a body, or the force with which gravity attracts it at latitude $45^{\circ}$. As to the usefulness of the equations, this has never heretofore been doubted. They have filled the engineer's need for a set of handy formulæ for accelerated motion, and they are easily understood by the student.

More than ten years ago a high-school student in despair over his problems in dynamics, on account of the obscurity of his text-book and of the teacher's explanations, appealed to the writer for assistance. He was told to forget the formulæ of the text-book, with its poundals and units of mass and to memorize the following: $V=\sqrt{2 g h}, S=1 / 2 V T, F T=$ $M V, F=M A, F S=1 / 2 M V^{2}$ and $M=W / g$, $F$ being force in pounds, $M$ nothing else than $W / g=W / 32.2$, or $W / 32.174$ (if great precision is needed) (no "concept of mass" needed) and $W$ weight in pounds (quantity of matter as weighed on a platform scale). With these equations the student soon solved every problem in the book that referred to bodies uniformly accelerated. Many times since the writer has had occasion to give the same advice, and always with the same result.

Here is a simple problem in acceleration, with its solution by the engineer's method and by the method of the committee's report.

What is the draw-bar pull required to accelerate a railroad car whose weight is 100,000 pounds, on a level track, in latitude $30^{\circ}$, at the rate of 1 foot per second per second, friction neglected? The engineer's solution: $F=M A, M=W / g, A=\left(V_{1}-V_{0}\right) \div T$

$F=100,000 \div 32.2 \times 1 / 1=3,105.6$ pounds.

The mathematician's solution: First look up the value of $g$ for latitude $30^{\circ},=32.131$. In latitude $45^{\circ} g=32.174$. Weight is the force with which gravity attracts a body at a given place. The unit of force (pound) is the force with which gravity attracts the standard pound at latitude $45^{\circ}$, where $g=32.174$. A weight of 100,000 pounds at latitude $30^{\circ}$ is therefore a force of $100,000 \times 32.131 / 32.174$ pounds, $=W$. " The formula $F=M A$ should never be used in the ordinary problems of en- 
gineering." Using the formula $F / W=A / g$ we have $F=A W / g$. Since $g$ is to be taken for latitude $30^{\circ}$ its value is 32.131. We then have $F=1 / 1 \times 100,000 \times 32.131 / 32.174 \times$ $1 / 32.131=3,108.1$ pounds.

If the engineer had used the more precise value of $g$ at latitude $45^{\circ}$ he would have obtained the same result as the mathematician. $\mathrm{He}$ would not consider that the value of $g$ at latitude $30^{\circ}$ entered into the problem at all. In the mathematician's solution it enters twice, in numerator and denominator, and therefore cancels out.

The only cases in which the engineer ever needs to consider the value of $g$ at latitudes other than $45^{\circ}$ are those of precise calculations in which the force of gravity at a particular place enters into the problem, as in the case of the velocity of falling bodies, the power of falling water, the value of the mechanical equivalent of heat, etc. Thus the mechanical equivalent of heat is 777.52 foot pounds, or the work of lifting 1 pound 777.52 feet high, at latitude $45^{\circ}$. The figure obtained by experiments made in raising weights at a lower latitude would be greater in the proportion that the attraction of gravity is less. Steam at a temperature of $327.8^{\circ} \mathrm{F}$. has a pressure of 100 pounds per square inch above vacuum if the pressure is measured by its lifting a weight (piece of metal) at latitude $45^{\circ}$. It would raise a heavier weight, in the ratio $32.174 / 32.131$, at latitude $30^{\circ}$, and a lever safety valve would have to be loaded with a larger weight at latitude $30^{\circ}$ than at latitude $45^{\circ}$ to resist the pressure. A bar of metal tested on a lever testing machine at latitude $45^{\circ}$ and showing an ultimate resistance of 32,131 pounds would show 32,174 pounds if tested at latitude $30^{\circ}$, since at latitude $30^{\circ}$ the poise on the lever would be attracted less by gravity than at latitude $45^{\circ}$, and it would have to be moved farther out on the scale beam to balance the ultimate load applied to the test piece.

Note on "The Concept of Mass" (Galley 35).

It is not clear just what the report means by the word "mass." It is defined in one paragraph as "the ratio $W / g$, or a quantity proportional to this ratio, $m=c(W / g), W$ here being a force and not a quantity of matter," but a little later appears the expression "the mass of the body measured in units of mass," indicating that by mass is meant quantity of matter.

A great deal of mental energy has been wasted by teachers and text-book writers in trying to give high school and college students a "concept of mass," and more trouble is yet in store for teachers and students if future text-books adopt the language of this report concerning the "concept."

A boy before he goes to the high school has a perfectly clear idea of matter and of weight. He knows that matter is weighed by the grocer on even-arm balances and platform scales, and he has seen meat weighed on spring balances. He knows that in order to answer the question "How much sugar is in that package?" the weight of the package is obtained by weighing it with a balance, using pieces of metal called weights, or by putting it on a spring balance and noting the indication. He knows that the weight of a pound of lead is 1 pound, whether it is weighed in London or in Panama, and that it is bought and sold as a pound everywhere that English weights are used. He has never heard the word "mass" except in its common meaning, of something like "bulk," or as a general term of indefinite quantity, as in the expression in the preface of the report, "lost sight of in a mass of details." When he begins to study physics, however, he has to learn that what he thinks he knows about weight is all wrong, that the weight of a thing is not constant, but variable, varying with the latitude and elevation above sea level; that it is not the measure of quantity of matter, but only of the force with which the earth's gravity attracts matter; that the word "mass" should be used where he formerly used "weight"; and that he must get a "concept" of mass different from the concept of weight. Later he learns that mass is also $c(W / g)$, the ratio of the force with which gravity attracts matter at a given place to the acceleration due to gravity at that place, multiplied by a coefficient, $c$, which has 
different values according to which system of units is used, there being six systems, kilogram-centimeter, pound-foot, dyne-centimeter, poundal-foot, $G_{0}$-kilogram-centimeter and $g_{0^{-}}$ pound-foot, that in the first system the value of $c$ is 980.665 ; in the second, 32.174 ; and in the other four systems 1, but that the last three are only in the text-books (and students must study them and pass examinations on them) and are never used in practise. $\mathrm{He}$ is also told that the engineer has a different unit of mass from the physicist, 32.2 pounds, which is not true.

When the student gets into practical engineering studies he is told to forget all he learned about the "concept of mass" and that he need think of the word "mass" only as a short term to use instead of the ratio $W / g$, $=M$, without any coefficient, $c$, that weight, $W$, has the same meaning that it has in commerce, quantity of matter, and that $g$ in that ratio is always the constant, 32.2, or, to be more precise, 32.174. What then is the use of confusing the young student with so many notions of the "concept of mass" when he has to unlearn them later?

\section{The Definitions that should be in the Text- books}

Criticisms of the definitions of mechanical units given in the report of the committee will fail of their proper effect unless other definitions are offered which the committee may possibly consider when the report is revised for final publication. The following definitions are offered for such consideration as they may deserve.

Weight, W. (1) Quantity of matter in a body, as weighed anywhere on an even balance scale with standard weights. (2) The force with which the earth's gravitation attracts a body at the sea level in latitude $45^{\circ}$ (or at any place where the acceleration due to gravity is 32.174 feet per second per second).

Unit of Weight. The pound, the quantity of matter in the standard piece of metal preserved in the bureau of standards in London.

Force. That which causes or tends to cause or to change motion. A push or a pull.
The Unit of Force. The pound; the attraction of the earth's gravitation on a pound of matter at the sea level at latitude $45^{\circ}$.

The weight of a body, $W$, is both the number of pounds of matter it contains and the number of pounds of force with which it is attracted to the earth at latitude $45^{\circ}$. The two numbers are exactly the same and therefore it is unimportant which definition is used in connection with the solving of problems.

Local weight, $W_{1}$, the force with which the earth's gravity attracts a body at any given place, measured in units of force. It may be determined by weighing it accurately on a spring balance which has been graduated at latitude $45^{\circ}$, with standard weights, or, more easily, by computation, multiplying the weight, $W$, by the ratio of the value of $g$ at the given locality to $32.174 . W_{1}$ varies with the location of a body. The difference between the weight of a body and the "local weight" at latitude $30^{\circ}$ is $(32.174 / 32.131)-1$, or 0.0013 of a pound, for each pound. The difference, 13 pounds in 10,000 , is so small that it need not be taken into account in any ordinary engineering calculation; in fact, the "local weight" is practically never used in engineering problems. When it is needed it is found by computation from the value of $g$ at the given locality.

Mass, $M$. (1) $W / g$, the ratio of the quantity of matter in a body (or of the attraction of the earth's gravity upon it at latitude $45^{\circ}$ ) to the acceleration due to gravity at latitude $45^{\circ}$ (32.174 feet per second per second). This is the meaning of the word "mass" when it is used in engineering problems. (2) The quantity of matter in a body, identical with $W$ (1) above, what is called weight ordinarily in commerce and in literature. This is the definition used in many text-books on physics, in which "weight" is restricted to mean what is defined as "local weight" above.

In answer to an objection that may be raised to the double definition of $W,(1)$ and (2), that the same word, in science, ought not to be used to express two ideas that are so dif- 
ferent as matter and force, it may be said that the two are in reality one definition. Suppose that a piece of metal, the standard pound, is hung on a spring balance. The position of the pointer on the scale is then marked 1 pound. A second piece of metal is substituted for the first, and if it brings the pointer to the same mark we say its weight is 1 pound. The 1 pound mark indicates two things at the same time, viz., that the quantity of matter in the second piece of metal is 1 pound, and that the force with which it is attracted by gravity is 1 pound. The word weight is thus logically and accurately defined by what may appear to be a double definition. No useful purpose is gained by applying another word "mass" to mean one part of this definition; on the contrary, the use of the word "mass" in this sense is the chief cause of all the confusion to which students are subjected in their study of dynamics.

\section{WILliam KeNT}

\section{SPECIAL ARTICLES}

\section{A STRENGTH AND ENDURANCE TEST}

Some time ago one of the newspapers in Kankakee, Ill., arranged a strength and endurance test in which the contestants were to walk 10 miles, each carrying on his back a sack of sand weighing 100 pounds. The course was laid out over the city streets around several blocks, and to complete the test a contestant must cover it 12 times. According to the estimate of the county surveyor, the course was 4,320 feet $(1,316.7$ meters) in length, and the 12 laps fixed upon for the contest would, therefore, give a total of 9 miles and 4,320 feet (15.8 kilometers), or approximately 93 miles, instead of the estimated 10 miles. The contest required about 4 hours, beginning about two o'clock in the afternoon and closing at dark, which would be about six o'clock on a November day. Prizes were offered to all who completed the test, and special prizes of smaller value to all who completed one or more rounds. The contest was a public affair, and was witnessed by a large gathering of people who lined the streets through which the contestants walked. A stand was arranged for the judges, and in every way provision seems to have been made to insure accuracy as regards entries, distance covered by each contestant, and so on.

Forty-eight men entered the contest. Of these 44 finished the first round, 25 the second round, 22 the third, 19 the fourth, 15 the fifth, 13 the sixth, 11 the seventh, 10 the eighth, 9 the ninth and 8 the tenth, while 6 completed the 12 rounds and fulfilled all the conditions of the contest.

The ages of the six successful contestants ranged from 21 to 52 years, four of them being 36 years old or over. Their body weight ranged from 150 to 255 pounds, the average being 189 pounds ( 86 kilograms). Of the 38 other contestants who completed one lap the ages ranged from 17 to 61 years, the majority being 30 years or over, while eight were over 40 years old. The body weights ranged from 120 to 200 , being on an average 162.4 pounds (74 kilograms).

As shown by the account of the contest published in the Kankakee press, each of the six men who completed the course felt that he was in condition to continue for a longer distance, but this the management did not permit.

The men who entered the contest were residents of Kankakee and were of different nationalities, including Germans, Scandinavians, French Canadians, a Pole and a Turk, while, judging by the published list of names, about one third of them were Americans. The newspaper ${ }^{1}$ under whose auspices the contest was held published a list of the winners, with names and addresses, and data regarding their age and weight, as well as a general description of the affair.

Through the courtesy of the editor of the paper, and by correspondence with a number of the contestants, including four of the six who completed the contest, additional data were secured, particularly with reference to the dietary habits of the men and their condition as regards training when they entered the contest. A circular letter of inquiry was sent to the successful contestants and to those 